\title{
Performance Evaluation of AODV and DSR routing protocols in MANET
}

\author{
Naresh Dobhal
}

\author{
Diwakar Mourya
}

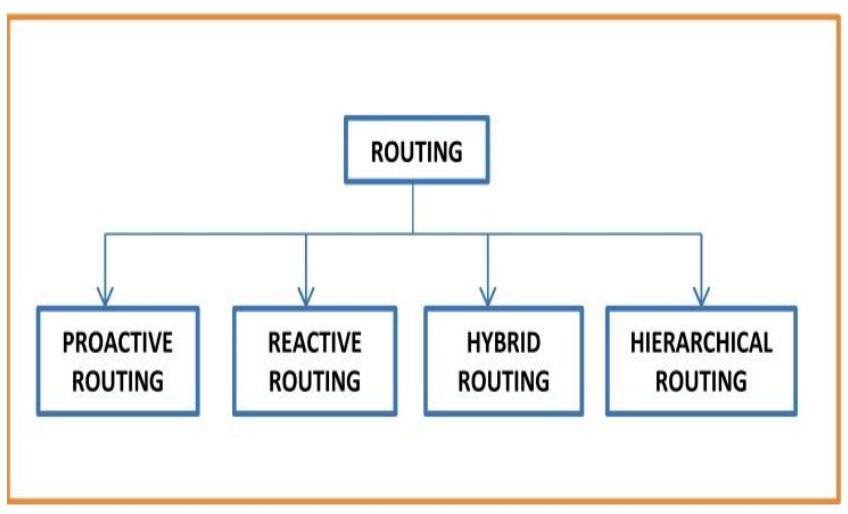

Figure 1: Types of routing in MANETs

a) Proactive routing protocols

These protocols maintain a routing table for entire nodes using the information present in the routing table of each individual node. Nodes periodically exchange topology information and maintain routes to various destinations even if they are not needed, which provides a minimal route selection time.

These protocols can be used in scenarios with:-

(i) Networks with lesser mobility of nodes.

(ii) Small network size with few nodes.

List of Proactive protocols are:-

i) DSDV Destination Sequenced Distance Vector routing protocol

ii) WRP Wireless routing protocol

iii) GSR Global State Routing protocol

iv) STAR Source Tree Adaptive Routing protocol

v) TBRPF Topology Broadcast Reverse Path Forwarding routing protocol

vi) OLSR Optimized Link State Routing protocol
vii) LANMAR
Landmark routing protocol

Advantages of Proactive protocols are:-

(i) Considerably lower route determination latency.

(ii) QoS guarantee related to connection setup or other real time requirements.

Disadvantages of Proactive protocols are:-

(i) High overhead on routing tables due to frequent routing updates.

(ii) Consumption of bandwidth for periodic updates. 
(iii) Maintaining of certain routes which may not be used even once.

\section{b) Reactive routing protocols}

These protocols are based upon the On-Demand Route Request approach in which nodes tend to find routes to destination nodes if there is a packet to be sent and its route is completely unknown at that time. The nodes using these protocols flood its neighbors with Route Request (RREQ) packets for computing a route to destination node.

These protocols can be used in scenarios with:-

(i) Networks with high mobility of nodes

List of Reactive protocols are:-

\begin{tabular}{|c|c|c|}
\hline i) & AODV & $\begin{array}{l}\text { Adhoc On-Demand Distance Vector } \\
\text { Routing protocol }\end{array}$ \\
\hline ii) & DSR & Dynamic Source Routing protocol \\
\hline & TORA & Temporally Ordered Routing algorithm \\
\hline & ABR & Associativity Based Routing protocol \\
\hline $\mathrm{y}$ & SSR & $\begin{array}{l}\text { Signal Stability based Adaptive Routing } \\
\text { protocol }\end{array}$ \\
\hline
\end{tabular}

Advantages of Proactive protocols are:-

(i) No overhead as routing information is obtained only when needed.

(ii) Scalability is possible as long as there is low mobility and less traffic.

Disadvantages of Proactive protocols are:-

(i) High route determination latency.

(ii) Flooding of RREQ packets can create congestion.

\section{c) Hybrid Routing Protocols}

Advantages of Proactive \& Reactive protocols are combined in Hybrid routing protocol approach. In this approach initially the routes to nearby nodes are maintained through some Proactive protocols while later on Reactive protocols can be used to discover the routes for far-away nodes or additionally activated nodes.

Hybrid routing protocols may present an optimal choice of path in different network scenarios.

ZRP (Zone Routing Protocol) is a hybrid routing protocol.

Underlying disadvantages of Hybrid routing approach are:-

(i) Usefulness of this approach requires the knowledge of nodes activated at any time.

(ii) Reaction to traffic demand depends upon the gradient of traffic volume.

\section{d) Hierarchical Routing protocols}

Scalability of Proactive \& Reactive protocols is limited due to their inherent designs. Enhancements made to these protocols improve performance but these enhancements still do not allow the protocol to scale well to larger networks.

Clustering protocols places the node into groups called Clusters and perform hierarchical routing between these clusters. This scheme increases the robustness of the routes by providing multiple possibilities for routing between clusters.

List of Reactive protocols are:-
(i) FSR Fisheye State Routing
(ii) CBRP Cluster Based Routing Protocol
(iii) $\begin{aligned} & \mathrm{ARC} \\ & \text { protocol }\end{aligned}$
(iv) DCA
Distributed Clustering Algorithm protocol
(v) DMAC
Distributed \& Mobility Adaptive Clustering algorithm

Advantages of Hierarchical Routing protocols are:-

(i) Hierarchy of nodes remains stable during mobility of nodes.

(ii) Flooding of control messages across the network are reduced greatly and only thee cluster leaders needs to be flooded.

Disadvantages of Hierarchical routing protocols are:-

(i) The depth of nesting of clusters \& addressing scheme reveals its advantages.

\section{DSR ROUTING PROTOCOL}

DSR (Dynamic Source Routing) is a simple yet efficient reactive routing protocol designed for multi-hop mobile adhoc networks. DSR protocol operates in two phases:-

(i) Route Discovery Phase

(ii) Route Maintenance Phase

Route discovery \& Route maintenance phase work in accordance for any possible route discovery \& maintenance to any random destination in the mobile adhoc network. DSR uses Source Routing mechanism in which any data packet that needs to be transferred carries the entire path in its header. This mechanism collects \& stores the addresses of each intermediate node traversed between the source node $\&$ the destination node.

\subsection{Route Discovery Phase}

Whenever a node $\mathrm{S}$ wants to send a data packet to a destination node $\mathrm{D}$, it initially checks its route cache for a possible path from node $\mathrm{S}$ to node $\mathrm{D}$ (from previously learned routes). If there exists no such path then source node $S$ may initiate a route discovery request otherwise existence of any such path (unexpired) in route cache is used in packet forwarding.

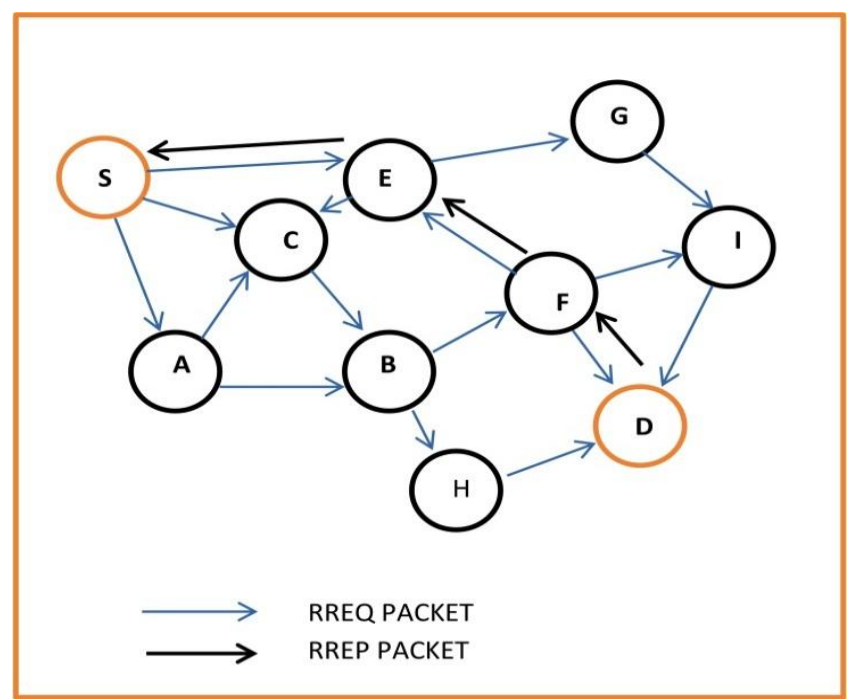

Figure 2:Route Discovery in adhoc network using DSR

A route discovery request is initiated by source node by broadcasting a single, local RREQ packet which is received approximately by all the neighboring nodes within the wireless vicinity of it. The RREQ packet contains information for identification of source node, destination node, set of intermediate nodes through which it has been forwarded (initially it is empty on creation) and a unique id set by source node. 
Any node that receives a RREQ packet would return a RREP (route reply) packet back to the source node if it is requested destination. RREP packet contains a copy of the route traversed by the RREQ packet. The source node $\mathrm{S}$ updates its route cache for the route received in RREP packet for further packet forwarding.

Else if this node has also received a copy of RREQ packet previously that originated from the same source containing the same request id or if the address of the node is already recorded in the packet, it simply discards the RREQ packet.

Else this node appends its address in the route record in RREQ packet and broadcasts it a local broadcast packet to nodes within its transmission capacity.

\subsection{Route Maintenance phase}

Each node transmitting a data packet also carries the responsibility for confirmation that the packet has been received at the next hop along the forward route. This can be easily accomplished using Active acknowledgement or passive acknowledgement [1].

If a packet if transmitted by some intermediate node for a maximum number of times and no confirmation is received, then this intermediate node returns a route error RERR packet back to the source node also indicating the link over which failure has occurred.

\subsection{Advantages of DSR}

(i) DSR is a reactive routing protocol which does not require flooding the network with route update messages.

(ii) Intermediate nodes (hops) can use route cache information efficiently to further reduce route discovery overheads.

(iii) Multiple routes to target node may get reported as intermediate nodes uses their local route cache for route discovery.

\subsection{Disadvantages of DSR}

(i) The size of packet header grows in size as the route length increases thereby reducing data carrying capability.

(ii) Route Reply Storm problem originates due to excessive route replies generated by the intermediate nodes using their local cache.

(iii) Collisions between route requests packets from neighboring nodes may occur.

\section{AODV ROUTING PROTOCOL}

AODV (Adhoc on-demand Distance Vector) is another ondemand (reactive) routing protocol widely used in mobile adhoc networks. AODV [4] incorporates some features of DSDV (Destination Sequenced Distance Vector) and DSR (Dynamic Source Routing) protocols. AODV protocol operates in two phases:-

$$
\text { Route Discovery Phase }
$$

$$
\text { Route Maintenance Phase }
$$

AODV and DSR are both reactive routing protocols yet they differ in their conceptual method of route discovery mechanism. DSR uses source routing whereas AODV uses hop-by-hop routing.

\subsection{Route Discovery Phase}

Whenever a source node $\mathrm{S}$ wants to send a data packet to a destination node $\mathrm{D}$, it initially checks it local routing table entries for a possible path to the aforesaid destination node. If an un-expired route is present in routing table entries it is chosen else route discovery phase is initiated by the source node by broadcasting a single local RREQ packet to the nodes within its transmission range.

The RREQ packet contains source node identification, destination node identification, destination sequence number assigned by the source node, broadcast identification, time to live. Destination sequence number is used to determine the newness (freshness/ up-to-dated) of the route. An intermediate node updates its route only if the destination sequence number of a current packet is greater than or equal to the destination sequence number stored at the node with smaller hop count.

Upon receiving a RREQ packet a node can return a RREP (route reply) packet back to the source node $\mathrm{S}$ if it is requested destination node $\mathrm{D}$ or if it knows a more recent up-to-date route to the destination (although the probability for such is low as compared to DSR protocol). RREP packet contains a copy of the route traversed by the RREQ packet.

Else if the node does not contain an entry for the requested route, it re-broadcasts the RREQ packet (RREQ packet gets rebroadcasted until it reaches the destination or time to live timer expires). The node also updates its routing table by recording the address of the intermediate node from which it first received this RREQ packet.

A node can discard a copy of RREQ packet if it has already been processed based on the destination sequence number (destination sequence number makes RREQ packets loop free $\&$ also enables the intermediate nodes to reply with latest information about routes)

\subsection{Route Maintenance Phase}

Nodes using AODV routing protocol periodically exchange beacons \& absence of a beacon within a stipulated period of time indicates a possible link failure.

Whenever a broken link is detected by any node while attempting to forward a data packet, it generates a route error RERR packet which is transmitted to all other nodes that might be using the broken link in their routing table entries. The RERR packet deletes all routing entries which are using the broken link. If a RERR message is received by a source node $S$, then it again initiates a RREQ packet.

\subsection{Advantages of AODV}

(i) Less delay in connection setup.

(ii) Latest and up-to-date routes can be discovered using the destination sequence number.

\subsection{Disadvantages Of Aodv}

(i) Routing table entries are purged (deleted) after a certain period of time even if any or some of the links are valid.

(ii) Periodic exchange of beacons for detecting broken links consumes bandwidth.

\section{SIMULATION OF MANET}

Extensive study of routing protocols in a variety of real time scenarios (environments) is required in obtaining a conclusion for using a better routing protocol for a given scenario. This extensive studies \& test may not be feasible at all times for a 
real time environment. However this restriction is eased with the help of special software called Simulators.Network simulators can effectively model hypothetical (conceptual) \& real time environments on a computer system. Now-a-days network simulators provide an easy-to-use, versatile and integrated GUI based designer tools for designing \& simulating networks. Some commercial (paid) network simulators are Qualnet, OPNET and some non-commercial (free) network simulators are ns2, ns3, Omnet++ [2].

\subsection{Simulation Setup}

For a detailed analysis of DSR and AODV routing protocols a non-commercial, discrete event simulator named as RiverBed Modeler Academic Edition 17.5.A. [5] is used. It is licensed academic simulator provided by RiverBed Corporation and is free only for educational purposes.

\subsection{Simulation Environment Parameters}

For a detailed investigation two different scenarios were created. The common parameters are:-

\begin{tabular}{|l|l|}
\hline No of nodes & 15 WLAN Workstations \\
\hline Other nodes & $\begin{array}{l}\text { Application configuration node } \\
\text { Profile configuration node } \\
\text { Mobility configuration node }\end{array}$ \\
\hline Area & $100 \mathrm{~m}^{*} 100 \mathrm{~m}$ \\
\hline $\begin{array}{l}\text { Application } \\
\text { used }\end{array}$ & High Load FTP \\
\hline Data Rate & $24 \mathrm{Mbps}$ \\
\hline Transmit power & $0.005 \mathrm{~W}$ \\
\hline Buffer size & 256000 \\
\hline Mobility speed & $50 \mathrm{~m} / \mathrm{s}$ \\
\hline Pause time & $0 \mathrm{~s}$ \\
\hline Start time & $10 \mathrm{~s}$ \\
\hline Stop time & End of simulation \\
\hline AODV \& DSR & Default parameters \\
\hline & \\
\hline Simulation time & 7200 seconds \\
\hline
\end{tabular}

\subsection{Simulation environment setup}

Simulation environment consists of 15 wireless mobile nodes which are placed uniformly along a grid to resemble a mobile adhoc network. These wireless nodes cover an area of $100 \mathrm{X}$ 100 meters with a mobility speed of 50 meters per second. Two different scenarios (exact replica of other) are made to run for 7200 seconds to simulate execution of AODV protocol and then DSR protocol for High Load FTP application session.

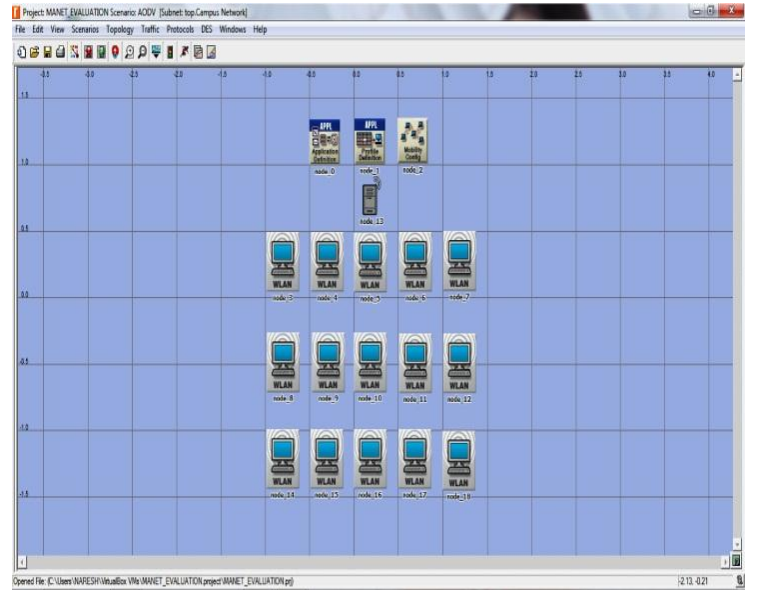

Figure 3: Setup of nodes in adhoc network

\section{AODV RESULTS}

The first scenario created in the mobile adhoc network simulated AODV as its routing protocol using 15 mobile stations. The parameters of AODV were set to default parameters (as set by RiverBed Modeler Academic Edition 17.5.A). The simulation setup was run for 7200 seconds and various statistics were collected from the GUI based result set provided by the network simulator. We collected three results:-

(a) Routing traffic received (bits/second)

(b) Routing traffic sent (bits/second)

(c) Total packets dropped

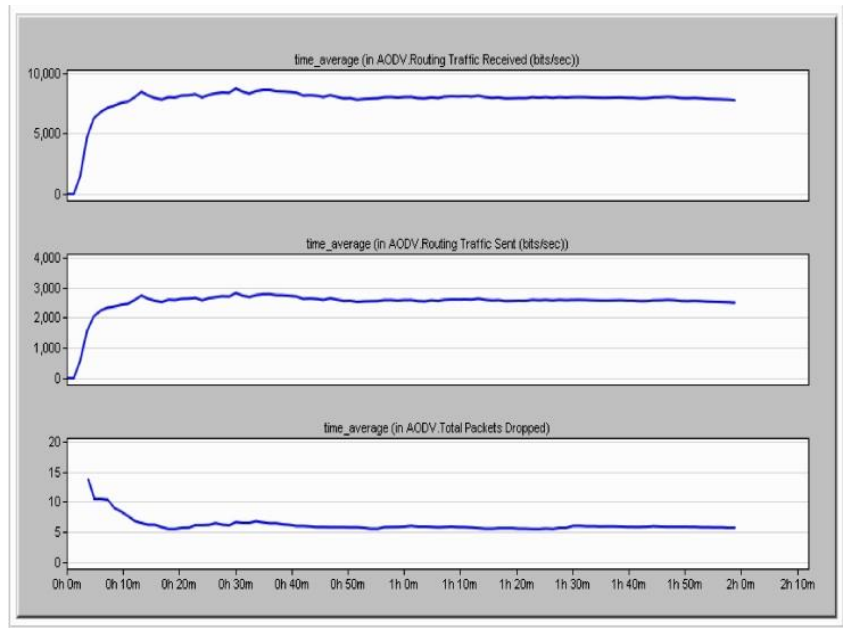

Figure 4: Results obtained by using AODV

\section{7. DSR RESULTS}

The second scenario created in the mobile adhoc network simulated DSR as its routing protocol. The parameters of DSR were again set to default parameters. We collected three results
(a) Routing traffic received (bits/second)
(b) Routing traffic sent (bits/second)
(c) Total packets dropped 


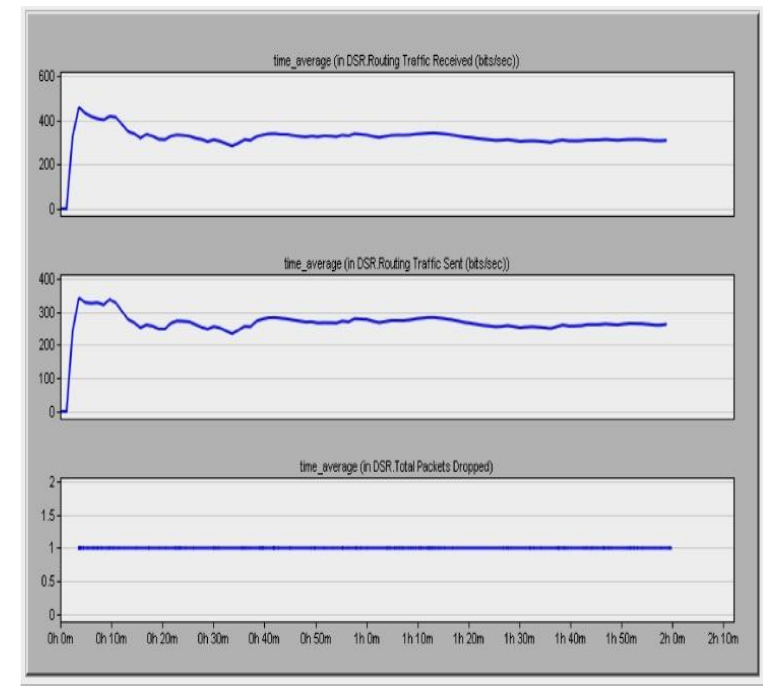

Figure 5 Results obtained by using DSR

\section{OVERLAID RESULTS}

For a better comparison an overlaid graph was prepared for
(a) Routing traffic received (bits/second)
(b) Routing traffic sent (bits/second)

This helps to simultaneously compare the parameters of AODV and DSR in a single graph. From the following data obtained it can be concluded that

(a) Routing traffic received in AODV ( approx. peak value $9000 \mathrm{bits} / \mathrm{s}$ ) is very large as compared to routing traffic received in DSR (approx. peak value 450 bits/s).

(b) Routing traffic sent in AODV ( approx. peak value $2800 \mathrm{bits} / \mathrm{s}$ ) is very large as compared to routing traffic received in DSR (approx. peak value 350 bits/s).

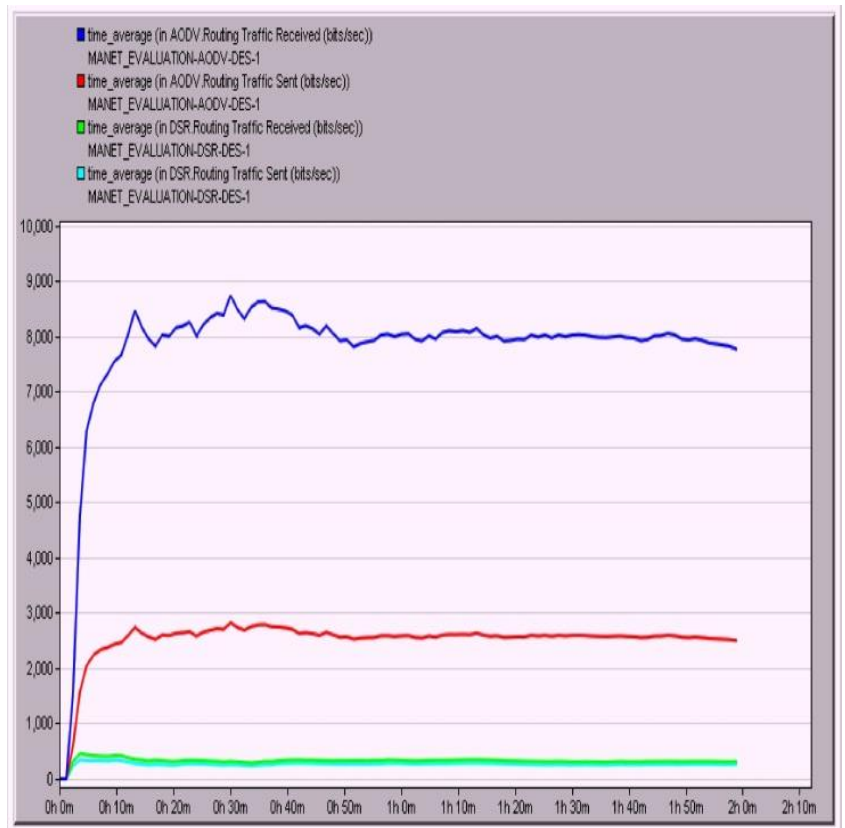

Figure 6: Overlaid result for AODV \& DSR

\section{QUALITY OF SERVICE RESULTS}

Results for QoS [3] were also taken into account for a better analysis. The following parameters were taken into account:-
(a) Delay
(b) Re-transmission
(c) Throughput

Delay represents the end-to-end delay of all the packets received by all nodes in the network. From the data available from simulation results it can be concluded that the end-to-end delay of packets from senders to receivers in very high in case of DSR as compared to AODV.

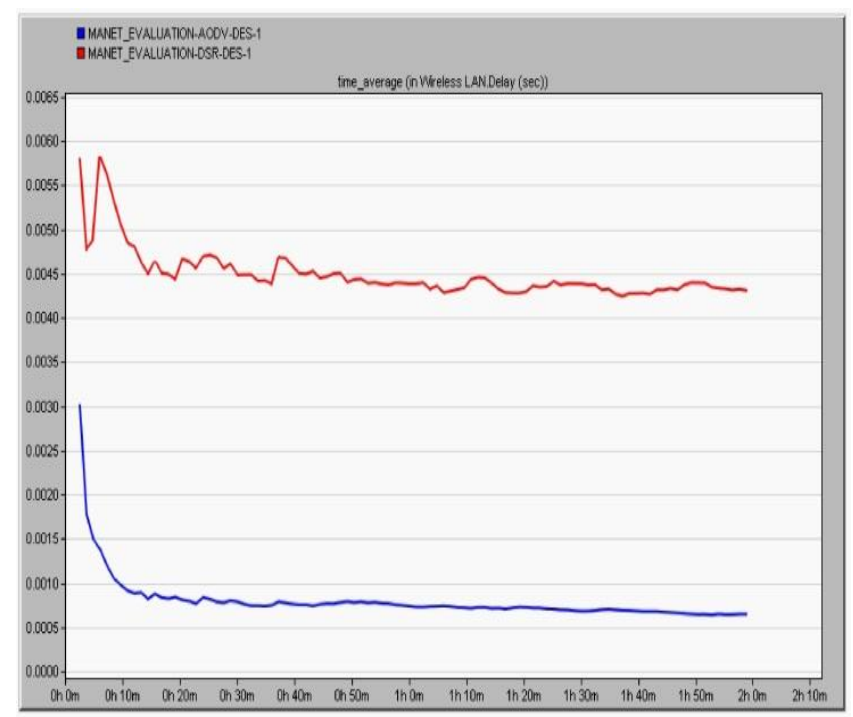

Figure 7: End-to-end delay

Re-transmission attempts are the numbers of attempts in the networks until the packet is successfully delivered to the destination node or it is discarded due to maximum number of retry attempts. It can be argued from the data obtained from the simulation that the number of re-transmission attempts in AODV is less compared as to DSR.

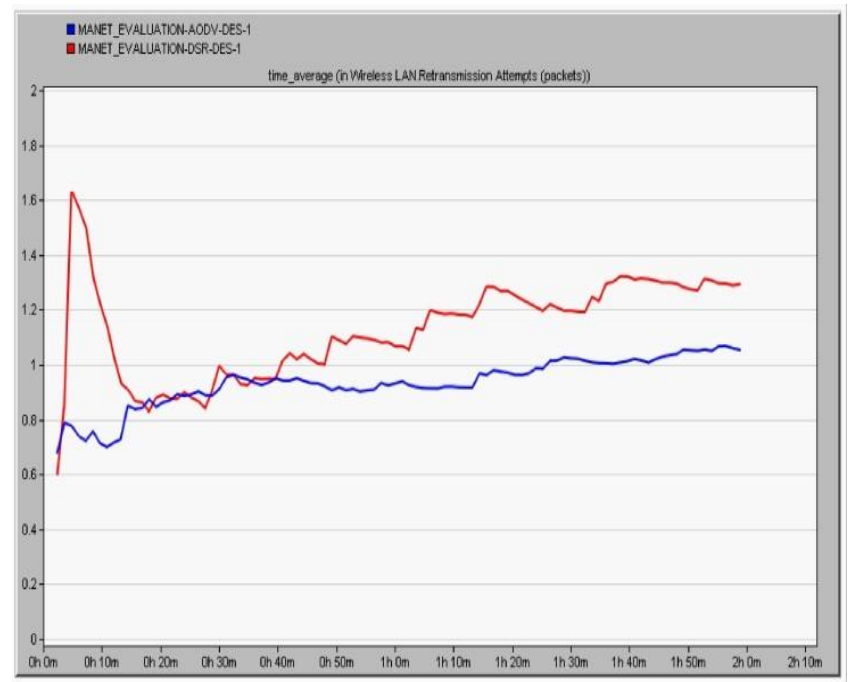

Figure 8:Re-transmission attempts

Throughput is the total number of bits delivered to higher layers of destination nodes. AODV again outperforms DSR in throughput which establishes its routing efficiency over DSR. 


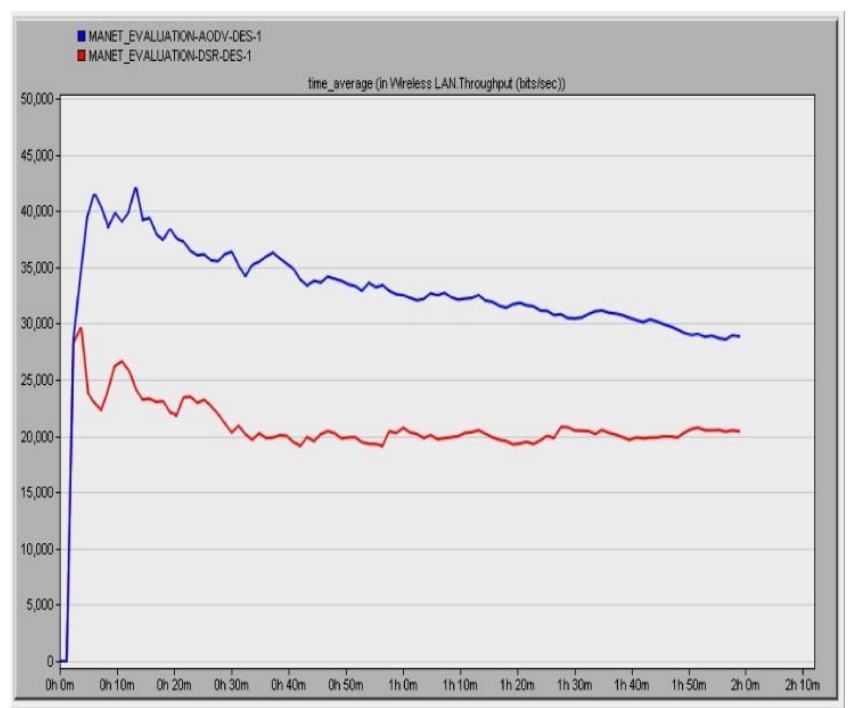

Figure 9 Throughput

\section{9 . CONCLUSION}

This paper compares the performance of two reactive routing protocols namely AODV and DSR. Some comprehensive simulated test results were presented for an adhoc network of about 15 nodes with a fixed mobility speed. Both protocols were analyzed against some specific parameters and it inferred that AODV routing protocol is efficient \& better suited for medium sized adhoc networks than DSR which is more effective in small sized adhoc networks.

\section{REFERENCES}

[1] John Jubin and Janet D. Tornow. "The DARPA PacketRadio Network Protocols."Proceedings of the IEEE, 75(1):21-32, January 1987.

[2] Mrs. Saba Siraj, Mr. Ajay Kumar Gupta, Mrs Rinku BadgujarIJARCCE [IISN : 2278 - 1021] Vol. 1 Issue 4 , June 2012.

[3] Nadia Qasim, Fatin Said, Hamid Aghvami. "Mobile adhoc Networking Protocols Evaluation through Simulation for Quality of Service".IAENG 36:1, IJCS_36_1_10

[4] C. E. Perkins, E. M. Royer, I. D. Chakeres, “Ad hoc OnDemand Distance Vector (AODV) Routing Protocol".draft-perkins-manet-aodvbis-00.txt, October 2003.

[5] RiverbedCorporation. Available athttp://www.riverbed.com. 